\title{
THE YOUNG OPEN CLUSTER BERKELEY 55*
}

\author{
Ignacio Negueruela AND Amparo Marco \\ Departamento de Física, Ingeniería de Sistemas y Teoría de la Señal, Universidad de Alicante, Apdo. 99, \\ E-03080 Alicante, Spain; ignacio.negueruela@ua.es, amparo.marco@ua.es \\ Received 2011 April 12; accepted 2011 November 8; published 2012 January 13
}

\begin{abstract}
We present $U B V$ photometry of the highly reddened and poorly studied open cluster Berkeley 55, revealing an important population of B-type stars and several evolved stars of high luminosity. Intermediate-resolution far-red spectra of several candidate members confirm the presence of one F-type supergiant and six late supergiants or bright giants. The brightest blue stars are mid-B giants. Spectroscopic and photometric analyses indicate an age $50 \pm 10$ Myr. The cluster is located at a distance $d \approx 4 \mathrm{kpc}$, consistent with other tracers of the Perseus Arm in this direction. Berkeley 55 is thus a moderately young open cluster with a sizable population of candidate red (super)giant members, which can provide valuable information about the evolution of intermediate-mass stars.
\end{abstract}

Key words: Galaxy: structure - open clusters and associations: individual (Berkeley 55) - stars: early-type - stars: evolution - supergiants

Online-only material: color figures, machine-readable and VO tables

\section{INTRODUCTION}

After exhaustion of $\mathrm{H}$ in their cores, stars evolve toward lower $T_{\text {eff }}$, becoming, according to their masses, red giants or supergiants (RSGs). Both high- and intermediate-mass stars are subject to complex physical processes in their later evolution, which result in important changes in their observable characteristics $\left(T_{\text {eff }}, L_{\text {bol }}\right)$. As a consequence, their evolutionary tracks trace loops in the H-R diagrams (e.g., Chiosi et al. 1992). The shape of these loops depends on the physics of the stellar interior, generally modeled via poorly understood parameters (e.g., Chiosi et al. 1992; Mowlavi \& Forestini 1994; Salasnich et al. 1999; Meynet \& Maeder 2000). The extent of semi-convection and overshooting has very important consequences on many aspects of stellar evolution, most notably the ratio of initial mass to white dwarf mass (e.g., Jeffries 1997; Weidemann 2000) and the boundary between stars that leave white dwarfs as remnants and those that explode as supernovae (SNe; e.g., Poelarends et al. 2008).

M-type supergiants represent the final evolutionary stage of moderately massive stars, with typical initial masses in the 8-25 $M_{\odot}$ range (Levesque et al. 2005). Such objects are the immediate progenitors of Type II-P SNe, the most frequent type of SN explosion in the local universe (Smartt 2009; Smith et al. 2011). Most of the explosions come from low-mass RSGs, stars with initial masses $M_{*} \lesssim 12 M_{\odot}$. The lower mass limit for a star to produce an $\mathrm{SN}$ has been estimated at $\approx 8.5_{-1.5}^{+1} M_{\odot}$ (Smartt et al. 2009). Nevertheless, stars with lower masses are also classified as supergiants, generally $\mathrm{K} \mathrm{Ib}$ objects, showing that the morphological separation between red supergiants and red giants does not coincide exactly with the boundary between high- and intermediate-mass stars.

Since stars become more luminous as they experience blue loops and later enter the asymptotic giant branch (AGB) branch, a given star may appear first as a bright giant and later in its evolution as a supergiant. Open clusters with large populations of evolved stars can help constrain the inputs of models and

\footnotetext{
* Partially based on observations collected at the Nordic Optical Telescope and the William Herschel Telescope (La Palma).
}

therefore improve our understanding of such basic questions. Identification and study of such clusters is thus an important astrophysical issue.

To this aim, we have analyzed a sample of poorly studied, cataloged, optically visible clusters in the Northern Hemisphere, using Two Micron All Sky Survey (2MASS) data (Skrutskie et al. 2006). We made use of the $Q_{\mathrm{IR}}$ index, a reddening-free parameter, defined as $(J-H)-1.70\left(H-K_{\mathrm{S}}\right)$, which has been proved to be a very useful tool for identification of intrinsically blue stars (see, e.g., Comerón \& Pasquali 2005; Negueruela \& Schurch 2007) and separation of luminous red stars from red dwarfs (Negueruela et al. 2011). Berkeley 55 (Be 55) was readily identified as containing an obvious clump of red luminous stars associated with a sequence of intrinsically blue stars.

Be 55 is a faint, compact open cluster in the constellation Cygnus. The WEBDA database ${ }^{1}$ provides coordinates R.A.: $21^{\mathrm{h}} 16^{\mathrm{m}} 58^{\mathrm{s}}$, decl.: $+51^{\circ} 45^{\prime} 32^{\prime \prime}\left(\ell=93^{\circ} 03, b=+1.80\right)$. Maciejewski \& Niedzielski (2007) presented $B V$ photometry of an extended field around the obvious cluster core and found it to be extremely compact, with $r_{\text {core }}=0.7$. They derived a distance $d=1.2 \mathrm{kpc}$ and an age $\log t=8.5$ (315 Myr), which was later used by Tadross (2008) to calibrate a fit to the 2MASS data in the region. However, the cluster looks too compact for this age and distance, while the isochrone fit of Tadross (2008) would suggest that most of the evolved cluster members are AGB stars, an extremely unusual situation.

In this paper, we present $U B V$ photometry of the cluster area and a spectroscopic survey of likely members, showing that its population is much younger than implied by the single-color fit of Maciejewski \& Niedzielski (2007). From our new data, we show that Be 55 is a young $(\log t \approx 7.7)$ open cluster with a rich population of evolved stars.

\section{OBSERVATIONS AND DATA}

\subsection{Optical Photometry}

$U B V$ photometry of Be 55 was obtained in service mode using ALFOSC on the Nordic Optical Telescope at the Roque

\footnotetext{
1 At http://www.univie.ac.at/webda/.
} 
Table 1

Log of the Photometric Observations Taken at the NOT on 2010 June for Berkeley 55

\begin{tabular}{lcc}
\hline \hline Berkeley 55 & $\begin{array}{c}\text { R.A. }=21^{\mathrm{h}} 16^{\mathrm{m}} 59^{\mathrm{s}} \mathrm{9} \\
(\mathrm{J} 2000)\end{array}$ & $\begin{array}{c}\text { Decl. }=+51^{\circ} 45^{\prime} 59^{\prime \prime} 0 \\
(\mathrm{~J} 2000)\end{array}$ \\
\hline Filter & \multicolumn{2}{c}{ Exposure times (s) } \\
\cline { 2 - 3 } & Long Times & Short Times \\
\hline$U$ & 900 & 250 \\
$B$ & 200 & 60 \\
$V$ & 40 & 10 \\
\hline
\end{tabular}

de los Muchachos Observatory (La Palma, Spain) on the night of 2010 June 14 . In imaging mode, the camera covers a field of $6.5 \times 6.5$ and has a pixel scale of $0{ }^{\prime} .19$ pixel $^{-1}$.

One standard field from the list of Landolt (1992), PG 1323-086, was observed twice during the night in order to provide standard stars for the transformation. Since the number of measurements was too low to trace the extinction during the night, we used the median extinction coefficients for the observatory, after verifying that the two measurements of the standards were fully compatible with these values. The images were processed for bias and flat-fielding corrections with the standard procedures using the CCDPROC package in IRAF. $^{2}$ Aperture photometry using the PHOT package inside DAOPHOT (IRAF, DAOPHOT) was developed on these fields with the same aperture, 21 pixels, for each filter.

Images of Be 55 were taken in two series of different exposure times to obtain accurate photometry for a magnitude range. The $\log$ of observations is presented in Table 1 . The reduction of the images of Be 55 was done with IRAF routines for the bias and flat-field corrections. Photometry was obtained by point-spread function (PSF) fitting using the DAOPHOT package (Stetson 1987) provided by IRAF. The apertures used are of the order of the FWHM. In this case, we used a value of 5 pixels for all images in the $U$ and $B$ filters and a value of 4 pixels for the $V$ filter images.

In order to construct the PSF empirically, we automatically selected bright stars (typically 25 stars). After this, we reviewed the candidates and discarded those that did not fulfill all the best conditions for a good PSF star. Once we had the list of PSF

2 IRAF is distributed by the National Optical Astronomy Observatories, which are operated by the Association of Universities for Research in Astronomy, Inc., under cooperative agreement with the National Science Foundation.
Table 3

Photometry for Stars in Berkeley 55

\begin{tabular}{lccccccr}
\hline \hline$N^{0}$ & $V$ & $\sigma_{V}$ & $(B-V)$ & $\sigma_{(B-V)}$ & $(U-B)$ & $\sigma_{(U-B)}$ & $N$ \\
\hline 1 & 18.218 & 0.038 & 1.541 & 0.028 & 0.842 & 0.013 & 2 \\
2 & 18.086 & 0.040 & 1.527 & 0.030 & 1.433 & 0.075 & 2 \\
3 & 19.400 & 0.024 & 1.768 & 0.030 & 1.361 & 0.080 & 1 \\
4 & 19.612 & 0.000 & 1.988 & 0.012 & 1.037 & 0.090 & 2 \\
5 & 19.274 & 0.011 & 1.732 & 0.034 & 0.976 & 0.024 & 2 \\
6 & 17.898 & 0.037 & 1.670 & 0.024 & 0.846 & 0.019 & 2 \\
7 & 19.769 & 0.029 & 1.912 & 0.040 & 1.027 & 0.091 & 1 \\
8 & 19.145 & 0.006 & 1.810 & 0.033 & 1.114 & 0.060 & 2 \\
9 & 16.891 & 0.021 & 1.393 & 0.041 & 0.580 & 0.011 & 4 \\
10 & 19.297 & 0.019 & 1.811 & 0.028 & 1.018 & 0.073 & 1 \\
\hline
\end{tabular}

(This table is available in its entirety in machine-readable and Virtual Observatory (VO) forms in the online journal. A portion is shown here for guidance regarding its form and content.)

stars $(\approx 20)$, we determined an initial PSF by fitting the best function between the five options offered by the PSF routine inside DAOPHOT. We allowed the PSF to be variable (in order two) across the frame to take into account the systematic pattern of PSF variability with position on the chip.

We needed to perform aperture correction for each frame in all filters. Finally, we obtained the instrumental magnitudes for all stars. Using the standard stars and the median extinction coefficients for the observatory, we carried out the transformation of the instrumental magnitudes to the standard system by means of the PHOTCAL package inside IRAF.

The number of stars that we could detect in all filters is limited by the long exposure time in the $U$ filter. We identify all stars with good photometry in all three filters on the image in Figures 1 and 2. In Table 2, we list their $X$ and $Y$ positions as seen in Figure 1, and their identification with objects in the 2MASS catalog. The designation of each star is given by the number indicated on the images (Figures 1 and 2). We have photometry for 237 stars in the field. In Table 3, we list the values of $V,(B-V)$, and $(U-B)$ with the standard deviation and the number of measurements for each magnitude or index.

We can compare our photometry with the only existing data set, that of Maciejewski \& Niedzielski (2007). We find average differences (literature - this work) in $V$ of $-0.1 \pm 0.3$. This shows important dispersion, but no systematic effects. The average difference in $(B-V)$ is $0.16 \pm 0.08$, suggesting a small systematic difference, though not much higher than the standard deviation. We note that, with a pixel size of 2 .'2 and a reported

Table 2

$(X, Y)$ Position on the Map of All Stars with $U B V$ Photometry, Together with Their 2MASS Identification and Their Coordinates

\begin{tabular}{llcccc}
\hline \hline$N^{0}$ & \multicolumn{1}{c}{$\begin{array}{c}Y \\
\text { (pixels) }\end{array}$} & $\begin{array}{c}Y \\
\text { (pixels) }\end{array}$ & $\begin{array}{c}\text { R.A. } \\
(\mathrm{J} 2000)\end{array}$ & $\begin{array}{c}\text { Decl. } \\
(\mathrm{J} 2000)\end{array}$ & $\begin{array}{c}\text { Name } \\
(2 \mathrm{MASS})\end{array}$ \\
\hline 1 & 15.28 & 1446.924 & 319.327021 & 51.790562 & $21171848+5147260$ \\
2 & 21.685 & 1886.522 & 319.326885 & 51.813904 & $21171845+5148500$ \\
3 & 30.195 & 943.208 & 319.3254 & 51.763935 & $21171809+5145501$ \\
4 & 31.361 & 531.363 & 319.325026 & 51.742191 & $21171800+5144318$ \\
5 & 33.537 & 991.763 & 319.325118 & 51.766521 & $21171802+5145594$ \\
6 & 43.785 & 1622.479 & 319.324686 & 51.799866 & $21171792+5147595$ \\
7 & 45.558 & 933.834 & 319.324094 & 51.763504 & $21171778+5145486$ \\
8 & 46.3 & 313.876 & 319.323648 & 51.73064 & $21171767+5143503$ \\
9 & 65.361 & 1177.859 & 319.322528 & 51.77639 & $21171740+5146350$ \\
10 & 79.822 & 608.989 & 319.320931 & 51.746292 & $21171702+5144466$
\end{tabular}

(This table is available in its entirety in machine-readable and Virtual Observatory (VO) forms in the online journal. A portion is shown here for guidance regarding its form and content.) 


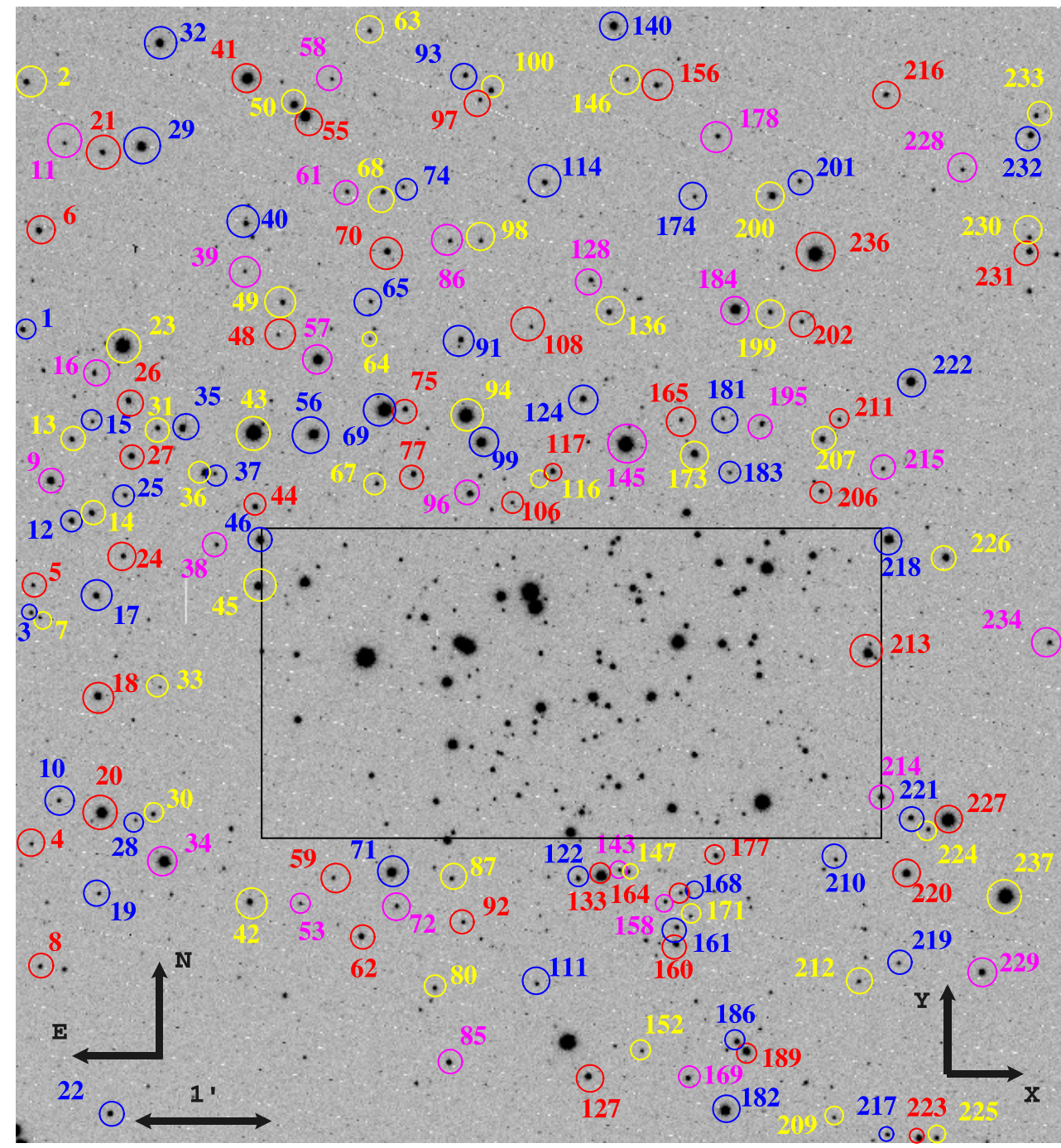

Figure 1. Finding chart for stars with photometry in the field of Berkeley 55. The image is one of our $V$-band frames. $X Y$ positions are listed in Table 2, where they are correlated to R.A. and decl. Stars inside the rectangle (which approximately defines the cluster core) are marked in Figure 2. Each star is identified by the nearest marker in the same color as the circle around it.

(A color version of this figure is available in the online journal.)

typical seeing around $5^{\prime \prime}$, the photometry of Maciejewski \& Niedzielski (2007) may be subject to important crowding effects near the cluster core.

\subsection{MASS Data}

We obtained $J H K_{\mathrm{S}}$ photometry from the 2MASS catalog. The completeness limit of this catalog is set at $K_{\mathrm{S}}=14.2$. None of the stars analyzed is bright enough to come close to the nonlinear regime for 2MASS. We used the 2MASS data to select targets for spectroscopy.

We selected our targets using 2MASS $J H K_{\mathrm{S}}$ data. We took a circle of radius $3^{\prime}$ around the cluster center and built the corresponding $K_{\mathrm{S}} /\left(J-K_{\mathrm{S}}\right)$ diagram (displayed in Figure 3). All the stars with $K_{\mathrm{S}} \leqslant 8.0$ (shown as diamonds) are clumped together in a small region of the diagram. Their $Q_{\mathrm{IR}}$ is $\approx 0.3$ in all cases. This shows that they are not field red dwarfs or red clump giants and suggests that they may be luminous red stars (Negueruela et al. 2011). We also used the $Q_{\mathrm{IR}}$ index to separate early-type stars (cf. Comerón \& Pasquali 2005; Negueruela \& Schurch 2007). As they are expected to show $Q_{\mathrm{IR}} \approx 0.0$, we select stars with $-0.15 \leqslant Q_{\mathrm{IR}} \leqslant 0.08$ (shown as filled circles and squares in Figure 3). This range is intended to account for the typical errors in 2MASS (generally $0.03-0.05 \mathrm{mag}$ in a given color for stars with $K_{\mathrm{S}}=12-13$ ) and also includes emissionline stars, which typically have $Q_{\mathrm{IR}} \lesssim-0.05$ (e.g., Negueruela et al. 2007). This selection clearly shows all early-type stars in the field to concentrate around $\left(J-K_{\mathrm{S}}\right) \sim 0.8$, forming an approximately vertical sequence with a sharp blue edge at $\left(J-K_{\mathrm{S}}\right) \sim 0.7$ (Figure 3).

We performed the same analysis for 2MASS data on concentric circles of increasing radii around the same position, finding no significant increase in the number of candidate members. This is in good agreement with the estimation by Maciejewski \& Niedzielski (2007) of a very concentrated cluster with $r_{\text {core }}=0.7$. Extending the search to $6^{\prime}$, we only find one candidate that seems to fall together with the red clump, both in the $Q / K_{\mathrm{S}}$ and $\left(J-K_{\mathrm{S}}\right) / K_{\mathrm{S}}$ diagrams. This is 2MASS $\mathrm{J} 21165198+5150410$, which we consider to be a candidate member below (under the name S61).

\subsection{Spectroscopy}

We obtained spectra in the far-red of the brightest early-type candidates (identified as green squares in Figure 3) and the seven candidate red luminous stars using the red arm of the 


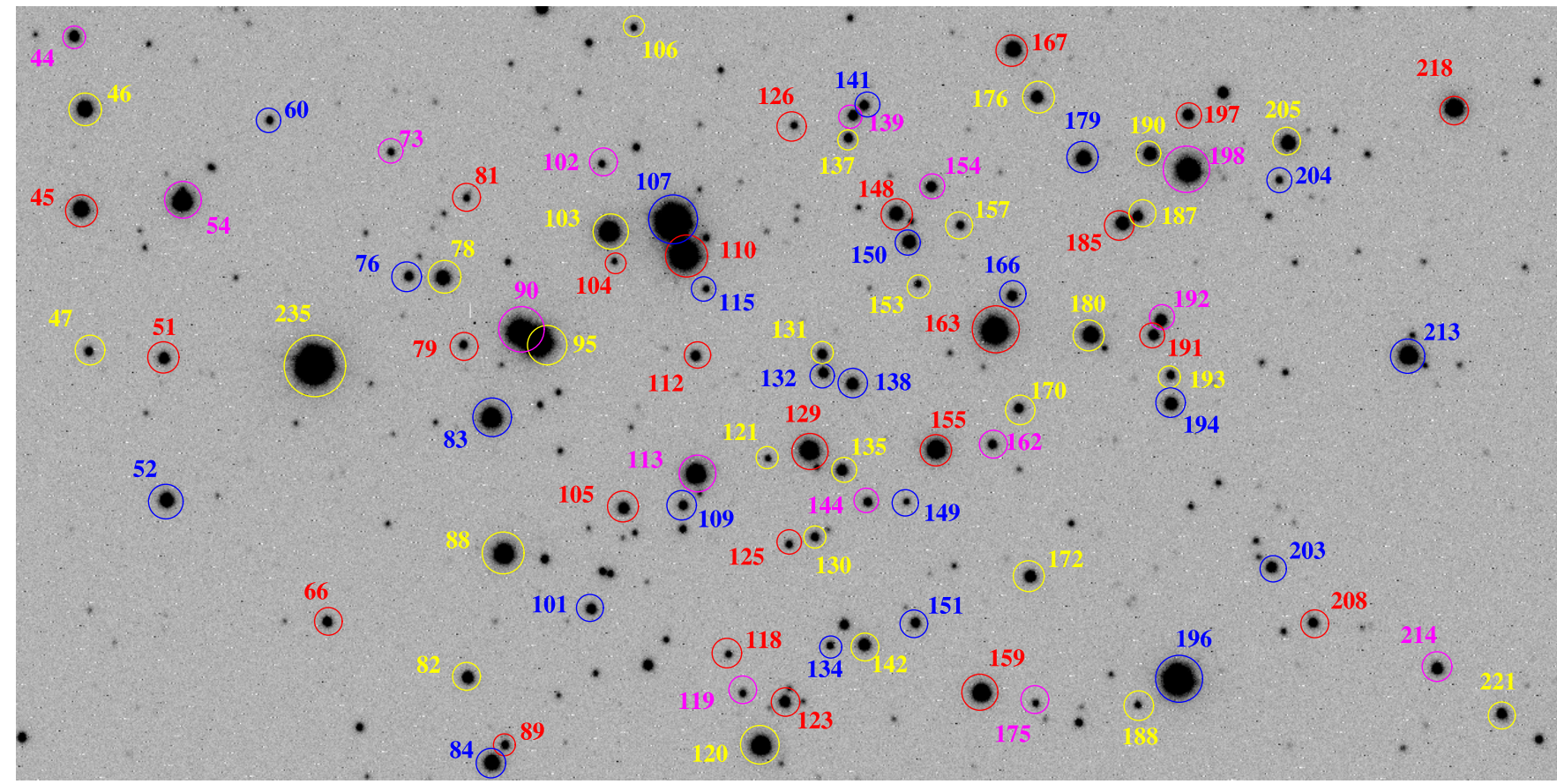

Figure 2. Finding chart for stars with photometry in the central part of Berkeley 55. The image is one of our $V$-band frames. $X Y$ positions are listed in Table 2, where they are correlated to R.A. and decl. Each star is identified by the nearest marker in the same color as the circle around it.

(A color version of this figure is available in the online journal.)

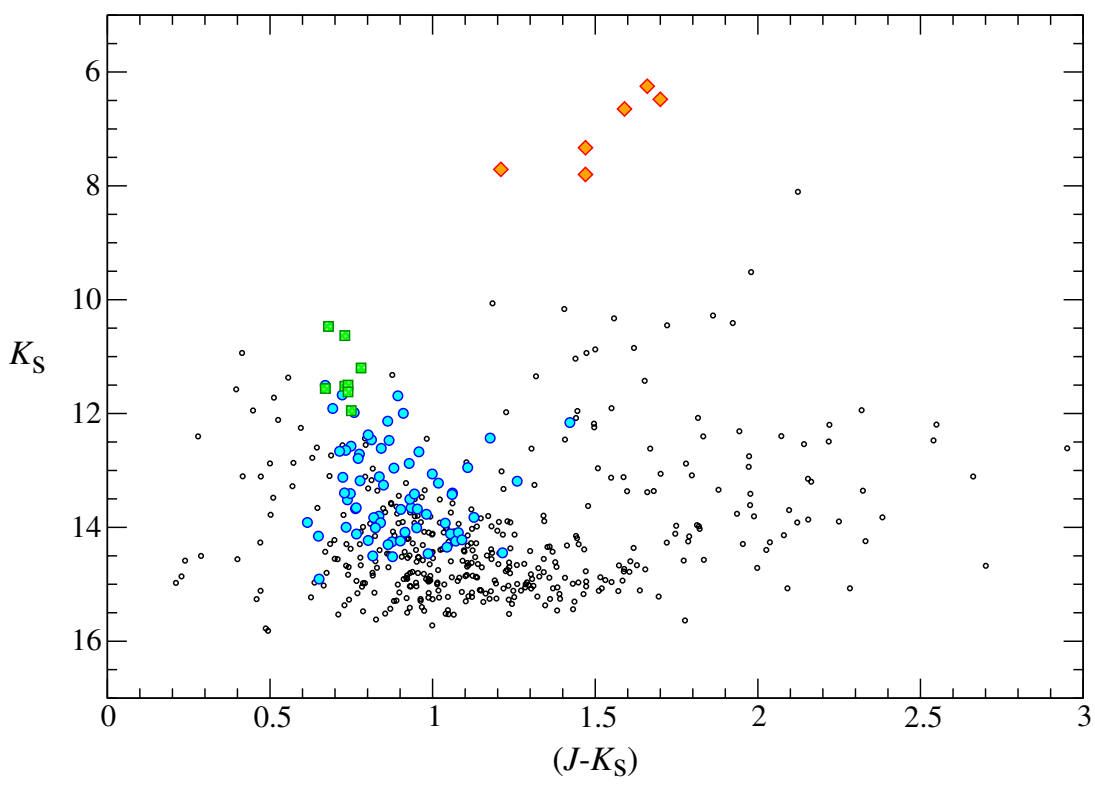

Figure 3. $K_{\mathrm{S}} /\left(J-K_{\mathrm{S}}\right)$ diagram for all stars in 2MASS within $3^{\prime}$ of the arbitrarily defined center of Be 55 . Stars selected as early-type stars based on their $Q_{\mathrm{IR}}$ index are marked as filled blue circles or green squares (if observed spectroscopically). The orange diamonds identify the clump of F-K supergiants.

(A color version of this figure is available in the online journal.)

ISIS double-beam spectrograph, mounted on the $4.2 \mathrm{~m}$ William Herschel Telescope (WHT) in La Palma (Spain). The instrument was fitted with the R600R grating and the Red+ CCD. This configuration covers the 7600-9000 A range in the unvignetted section of the CCD with a nominal dispersion of $0.5 \AA$ pixel $^{-1}$. Observations were taken during a service night on 2007 July 26 and then completed during a run on 2007 August 21. In July, the CCD was unbinned and a 1".5 slit was used. In August, the CCD was binned by a factor of two in the spectral direction and a $1^{\prime \prime} .2$ slit was used. In both cases, the resolution element is expected to be $\sim 4$ unbinned pixels. This has been checked by measuring the width of arc lines, which is on average $\approx 2.1 \AA$ for both configurations. The resolving power of our spectra is therefore $R \sim 4000$.

A $\log$ of these observations is presented in Table 4, where each star is given an identification starting with $\mathrm{S}$, and the correspondence with the numbering in the photometry is indicated. In addition to the stars targeted, two other photometric members also fell inside the slit by chance. One of them, no. 213 (S19 in Figure 8), is a bright member which turns out to be a Be star. The other one, no. 170, is very faint and the spectrum is noisy, simply confirming that it is a B-type star. 
Table 4

Log of Spectroscopic Observations

\begin{tabular}{|c|c|c|c|c|c|}
\hline Star & Phot $^{\mathrm{a}}$ & $\begin{array}{l}\text { Exposure } \\
\text { Time (s) }\end{array}$ & Date & $\begin{array}{l}\text { Time } \\
\text { (UT) }\end{array}$ & $\mathrm{S} / \mathrm{N}^{\mathrm{b}}$ \\
\hline $\mathrm{S} 1$ & $\ldots$ & 200 & 2007 Aug 21 & 01:07 & 300 \\
\hline $\mathrm{S} 2$ & 196 & 400 & $2007 \mathrm{Jul} 26$ & $00: 43$ & 340 \\
\hline S3 & 163 & 400 & 2007 Jul 26 & $00: 43$ & 220 \\
\hline $\mathrm{S} 4$ & 110 & 400 & 2007 Jul 26 & 01:12 & 260 \\
\hline S5 & 107 & 400 & 2007 Jul 26 & $01: 12$ & 310 \\
\hline S6 & 145 & 400 & 2007 Jul 26 & $00: 58$ & 330 \\
\hline S61 & $\ldots$ & 200 & 2007 Aug 21 & $01: 13$ & 370 \\
\hline S7 & 94 & 400 & 2007 Jul 26 & 01:12 & 140 \\
\hline S8 & 99 & 1000 & 2007 Aug 21 & $01: 27$ & 110 \\
\hline S9 & 198 & 400 & 2007 Jul 26 & $00: 58$ & 110 \\
\hline S10 & 83 & 1000 & 2007 Aug 21 & $01: 27$ & 140 \\
\hline $\mathrm{S} 12$ & 113 & 600 & 2007 Jul 26 & $01: 24$ & 90 \\
\hline $\mathrm{S} 13$ & 129 & 600 & $2007 \mathrm{Jul} 26$ & $01: 24$ & 90 \\
\hline $\mathrm{S} 15$ & 159 & 1000 & 2007 Aug 21 & 02:01 & 90 \\
\hline S16 & 133 & 1000 & 2007 Aug 21 & 02:01 & 100 \\
\hline
\end{tabular}

Notes.

a Cross-correlation with numbering system in Table 3.

b The $\mathrm{S} / \mathrm{N}$ has been estimated for the B-type stars from the rms of fits to continuum regions. In the red supergiants, where there are no spectral windows free of lines, it is an extrapolation based on the count rate. The $\mathrm{S} / \mathrm{N}$ is for each $0.5 \AA$ Ainned pixel.

All spectroscopic data were reduced using the Starlink software packages CCDPACK (Draper et al. 2000) and FIGARO (Shortridge et al. 2004). We used standard procedures for bias subtraction and flat-fielding (with internal halogen lamps). The spectra have been normalized to the continuum using DIPSO (Howarth et al. 1998).

\section{RESULTS}

\subsection{Observational H-R Diagram}

We start the photometric analysis by plotting the $V /(B-V)$ and $V /(U-B)$ diagrams for all stars in the field. In Figure 4, we can observe that the cluster sequence (as defined by the spectroscopic members) is heavily contaminated by what seems to be foreground population.

\subsubsection{The Reddening Law}

The first step is determining whether the extinction law in the direction of the field is standard. We start by selecting the B-type stars observed spectroscopically. For them, we calculate the ratio $X=E(U-B) / E(B-V)$ using the calibration of intrinsic colors by Fitzgerald (1970). For six stars, we find rather homogeneous values, with average $X=0.67 \pm 0.03$, quite close to the standard value 0.72 .

We also use the CHORIZOS $\left(\chi^{2}\right.$ code for parameterized modeling and characterization of photometry and spectroscopy) code developed by Maíz-Apellániz (2004). This code fits synthetic photometry derived from the spectral distribution of a stellar model convolved with an extinction law (Cardelli et al. 1988 ) to reproduce the observed magnitudes. We use our $U B V$ photometry and $J H K_{\mathrm{S}}$ data from 2MASS. This set of photometric values is well suited to find the general shape of the extinction law (Maíz-Apellániz 2004). On the other hand, the functional form of the law used by Cardelli et al. (1988), a seventh-degree polynomial, may lead to an artificial bumpiness of the extinction law for high values of $E(B-V)$.

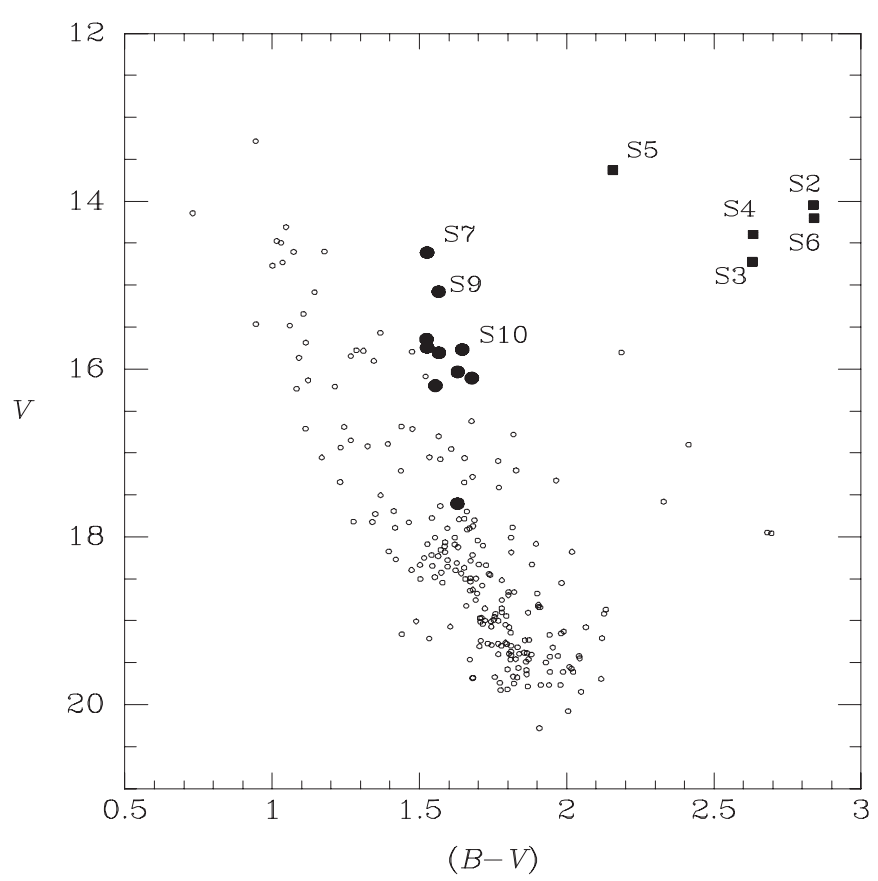

Figure 4. $V /(B-V)$ diagram for all stars in the field of Be 55. Filled circles represent B-type stars observed spectroscopically and filled squares are supergiant stars.

For the stars with spectral types, we use as input for CHORIZOS the $U B V J H K_{\mathrm{S}}$ photometry and the $T_{\text {eff }}$ corresponding to the spectral type derived according to the calibrations of Fitzgerald (1970). The output of CHORIZOS is the value of $R$ and the color excess $E(B-V)$. For all our stars, the preferred value of $R$ is close to 3.1, with little dispersion.

\subsubsection{Membership Determination}

Since the extinction is close to standard, we can use the classical $Q$ parameter (Johnson \& Morgan 1952) to estimate the spectral types for all the stars in the field. A first test with the stars with spectral types supports the use of $Q=$ $(U-B)-0.67(B-V)$, as the standard value 0.72 gives too early spectral types when compared with the spectroscopic ones (see below). Even such small difference in the ratio of color excesses, when combined with the high $E(B-V)$, introduces differences of about two subtypes.

Taking into account the photometric spectral types and the position of the stars in the color-magnitude diagrams (CMDs), we select the likely members of Be 55 . We identify 138 members, all with spectral types between $\mathrm{b} 3$ and $\mathrm{a} 0$. We proceed then to estimate the reddening for all the likely members with CHORIZOS. As input, we use the $U B V J H K_{\mathrm{S}}$ photometry and the $T_{\text {eff }}$ corresponding to the photometric spectral type, according to the calibrations of Fitzgerald (1970). We find that the average color excess is $E(B-V)=1.85 \pm 0.16$, where the error represents the dispersion in individual values among all 138 members in our photometric field.

In Figure 5, we plot the dereddened $M_{V} /(B-V)$ diagram for likely members of $\mathrm{Be} 55$. We perform a visual fit to the zero-age main sequence (ZAMS) from Mermilliod (1981) and from Schmidt-Kaler (1982), obtaining a distance modulus $\mathrm{DM}=13.0 \pm 0.3$. In Table 5, we display the values of $E(B-V)$, $V_{0}$, and photometric spectral types for all photometric members. 


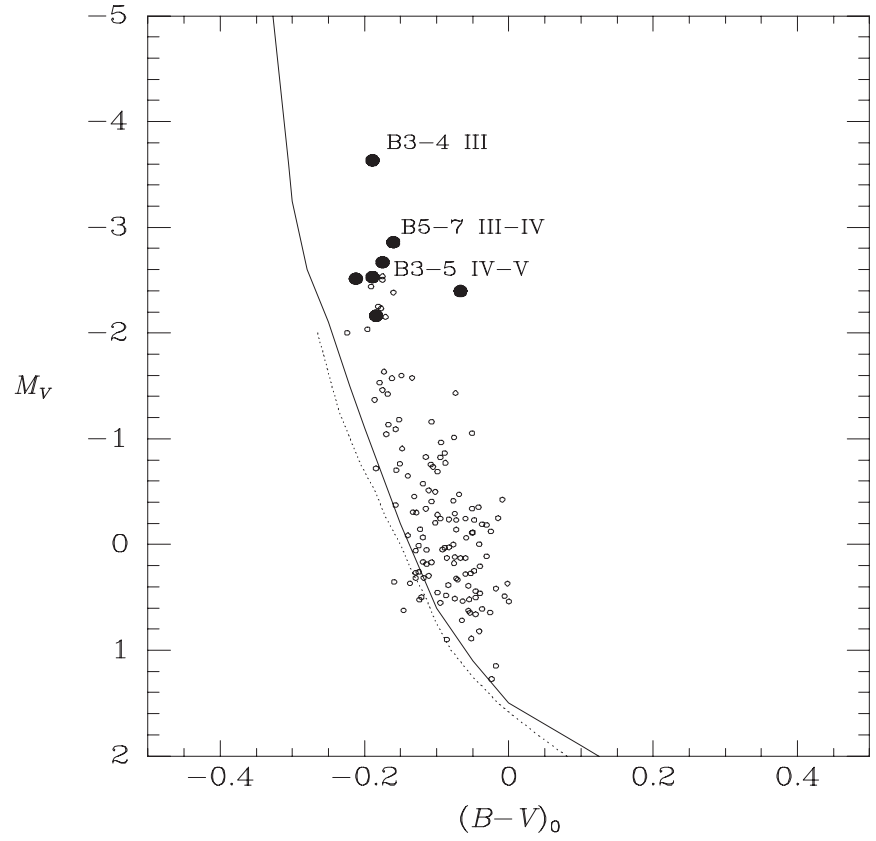

Figure 5. Dereddened $M_{V} /(B-V)$ diagram for likely members of Be 55 . The dashed line shows the ZAMS from Mermilliod (1981), and the solid line, the ZAMS from Schmidt-Kaler (1982). Filled circles indicate stars with spectra. The spectral types of the brightest members are shown. Note that the Be shell star S9 is not selected as a likely member because of its anomalous colors.

\subsection{Spectroscopic Analysis}

Because of the high reddening, stars in Berkeley 55 are faint in $B$ and intermediate-resolution spectra in the classification
Table 5

Photometry and Reddening for Stars Selected as Likely Members of Berkeley 55

\begin{tabular}{lccccc}
\hline \hline Star & $\begin{array}{c}E \\
(B-V)\end{array}$ & $\sigma_{E(B-V)}$ & $V_{0}$ & $\sigma_{V_{0}}$ & $\begin{array}{c}\text { Photometric } \\
\text { Spectral Type }\end{array}$ \\
\hline 1 & 1.67 & 0.03 & 13.26 & 0.06 & $\mathrm{~b} 8$ \\
4 & 2.15 & 0.02 & 13.36 & 0.07 & $\mathrm{~b} 7$ \\
5 & 1.76 & 0.02 & 14.27 & 0.11 & $\mathrm{~b} 9$ \\
6 & 1.74 & 0.03 & 12.53 & 0.04 & $\mathrm{~b} 8$ \\
7 & 1.99 & 0.02 & 13.32 & 0.09 & $\mathrm{~b} 8$ \\
8 & 1.89 & 0.02 & 13.51 & 0.07 & $\mathrm{~b} 9$ \\
10 & 1.94 & 0.02 & 13.52 & 0.07 & $\mathrm{~b} 8$ \\
11 & 1.84 & 0.02 & 13.46 & 0.07 & $\mathrm{~b} 9$ \\
13 & 1.6 & 0.02 & 13.89 & 0.08 & $\mathrm{~b} 8$ \\
15 & 1.74 & 0.02 & 13.65 & 0.1 & $\mathrm{~b} 9$
\end{tabular}

Note. ${ }^{\text {a }}$ The two known Be stars, S9 (no. 199) and S19 (no. 213) have been left out, as their intrinsic color cannot be calculated.

(This table is available in its entirety in machine-readable and Virtual Observatory (VO) forms in the online journal. A portion is shown here for guidance regarding its form and content.)

region would require long integrations with large apertures. Alternatively, we resort to the far-red, where the stars are much brighter and classification criteria exist.

\subsubsection{Bright Red Stars}

Figure 6 shows the $z$-band spectra of the seven bright red stars in the field preselected as candidate members. Six of them have fairly similar spectra, typical of late-type stars, with S5 appearing clearly earlier.

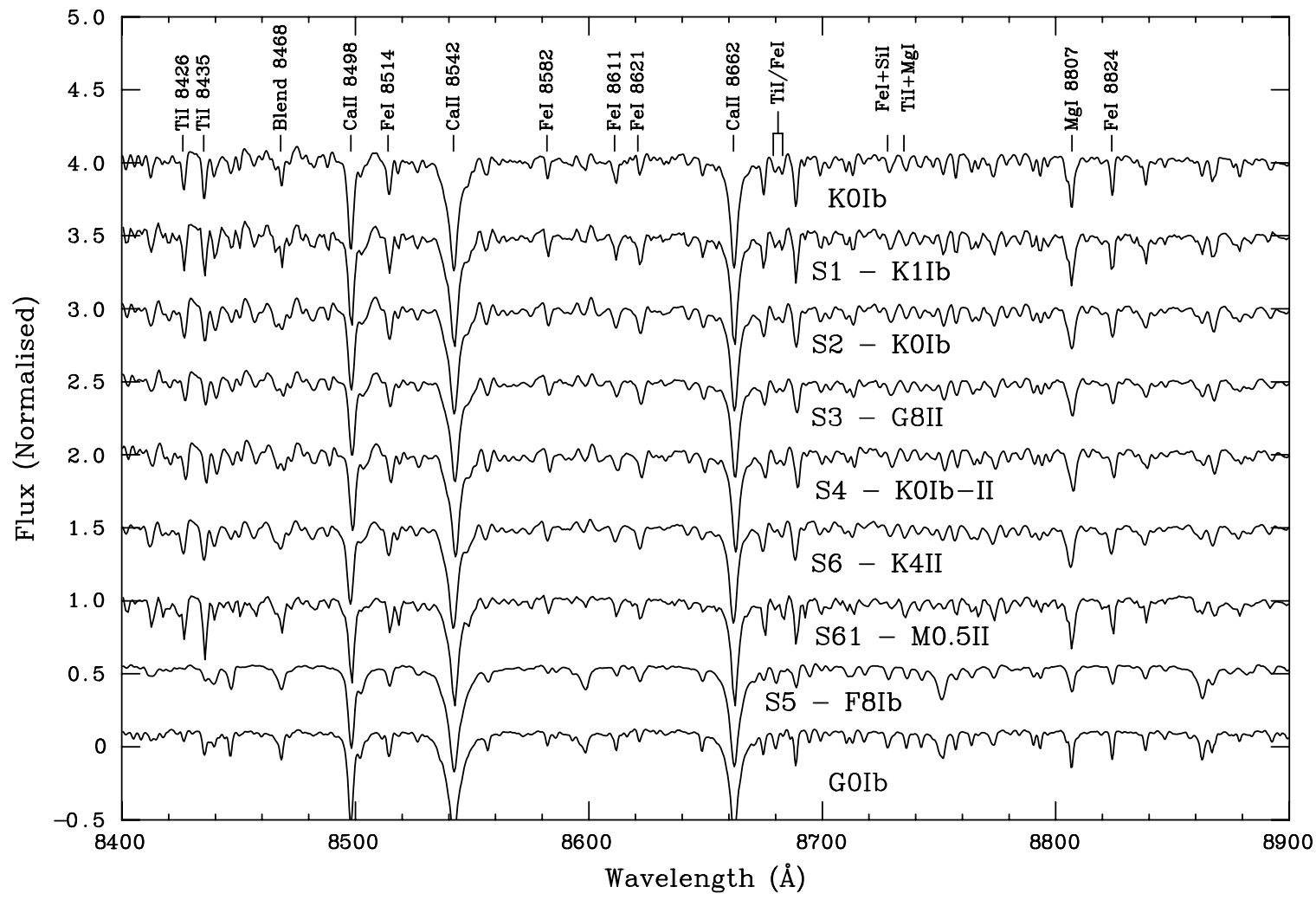

Figure 6. $z$-band spectra of seven bright red stars in the field of Be 55, together with two reference stars from the Indo-US library (Valdes et al. 2004). The main temperature criterion for G-K luminous stars in this range is the Ti I $8683 \AA / \mathrm{Fe}$ I $8679 \AA$ A ratio. The strength of the metallic spectrum is the main criterion to determine luminosity at a given spectral type, with the Ti I/Fe I blend at $8468 \AA$ showing a pronounced dependence. S5 has a much earlier spectral type and the Paschen lines are still seen. The top spectrum corresponds to HD 207089; the bottom one to HD 204867 (both classified in Keenan \& McNeil 1989). 
Table 6

Measured Equivalent Widths ${ }^{\text {a }}$ (in $\AA$ ) for the Main Quantitative Luminosity Indicators for Red Luminous Stars in Be 55

\begin{tabular}{lcccc}
\hline \hline Star & Ca II $8498.0 \AA$ & Ca II 8542.1 & Ca II 8662.1 & Blend 8468 \\
\hline S1 & 3.2 & 5.2 & 4.0 & 1.4 \\
S2 & 3.0 & 5.3 & 4.0 & 1.3 \\
S3 & 2.7 & 4.9 & 3.6 & 1.0 \\
S4 & 3.6 & 5.0 & 3.6 & 1.2 \\
S5 & 2.9 & 6.0 & 4.9 & $\ldots$ \\
S6 & 2.8 & 4.9 & 3.5 & 1.2 \\
S61 & 2.4 & 4.7 & 3.7 & 1.1 \\
\hline
\end{tabular}

\section{Notes.}

${ }^{a}$ Because of the difficulty in determining the continuum in red stars, uncertainties may be estimated at $\pm 0.3 \AA$ for each Ca II line and $\pm 0.1 \AA$ for the $8468 \AA$ blend.

b Note that the values for S5 must include the blended Paschen lines.

In late-type stars, at a given metallicity, the strength of the metallic spectrum increases with luminosity (Ginestet et al. 1994; Carquillat et al. 1997). Luminosity criteria at resolutions similar to ours are discussed in Negueruela et al. (2011). In particular, the strength of the Ca II triplet is strongly correlated with luminosity (Díaz et al. 1989; Zhou 1991), though it also depends on metallicity and spectral type. The intensity of the Ca II triplet lines (see measurements in Table 6) shows without doubt that all of our seven objects are luminous stars.

The O I $7774 \AA$ triplet is seen in stars earlier than late G. Of all of our bright red stars, S5 is the only one displaying this feature. On the other hand, none of our stars displays TiO bands in the spectral range measured, showing that they are earlier than $\sim$ M1 (e.g., Negueruela et al. 2011). For luminous stars in the G5-K5 range, the ratio between the Ti I $8683 \AA$ and the Fe I $8679 \AA$ lines is very sensitive to temperature (Carquillat et al. 1997). By using this criterion, we find all the stars to lie between G8 and K4, with the exception of S61, which is slightly later, though it is still earlier than M1, as the TiO band head at $8660 \AA$ is hardly detectable.

For spectral types G-K, Díaz et al. (1989) find that stars with a combined equivalent width (EW) for the two strongest Ca II lines, EW(Ca II 8542+Ca II 8662) > $9 \AA$, are definitely supergiants, independent of metallicity or spectral type, though some supergiants may present slightly lower values of EW. This has recently been re-assessed for a larger sample (Negueruela et al. 2011). Attending to all the criteria and to direct comparison to the standards, S1 (2MASS J21165840+5743262, saturated in our photometry) and S2 fall clearly in the supergiant region, though both have low luminosity. All the other objects lie in the region where both supergiants and bright giants are found. Attending to their morphology, we classify them as bright giants, with S4 closer to being a supergiant than the others. The spectral types finally derived are listed in Table 7.

In the case of S5, comparison to MK standards observed at similar resolution (e.g., Cenarro et al. 2001) suggests that it is an F8 supergiant. The combined EW of the two strongest Ca II lines is close to $11 \AA$, but the Paschen lines are still present at this spectral type and thus $\mathrm{Pa} 13$ and $\mathrm{Pa} 15$ must be blended with $\mathrm{Ca}$ II $8662 \AA$ and Ca II $8542 \AA$, respectively. By measuring the EWs of $\mathrm{Pa} 12, \mathrm{~Pa} 14$, and $\mathrm{Pa} 17$, we estimate that they contribute at most $1.5 \AA$ to the blend. Therefore, S5 is a supergiant according to all calibrations of the Ca II triplet (Díaz et al. 1989; Mallik 1997; Zhou 1991). The calibration of $M_{V}$ against the EW of the O I $7774 \AA$ triplet (Arellano Ferro et al. 2003) indicates for this object $M_{V}=-3.7$, with a large uncertainty. This is consistent
Table 7

Derived Parameters for Stars with Spectroscopy

\begin{tabular}{llrccc}
\hline \hline ID & \multicolumn{1}{c}{$\begin{array}{c}\text { Spectral } \\
\text { Type }{ }^{\text {b, }}\end{array}$} & $K_{\mathrm{S}}{ }^{\mathrm{a}}$ & $\left(J-K_{\mathrm{S}}\right)$ & $(J-K)_{0}$ & $\begin{array}{c}E \\
\left(J-K_{\mathrm{S}}\right)\end{array}$ \\
& \multicolumn{1}{c}{ K1 Ib } & 6.25 & 1.66 & 0.68 & 0.98 \\
S1 & K0 Ib & 6.65 & 1.59 & 0.63 & 0.96 \\
S2 & 7.80 & 1.47 & 0.56 & 0.91 \\
S3 & G8 II & 7.33 & 1.47 & 0.63 & 0.84 \\
S4 & K0 Ib-II & 7.71 & 1.21 & 0.35 & 0.86 \\
S5 & F8 Ib & 6.48 & 1.70 & 0.90 & 0.80 \\
S6 & K4 II & 6.15 & 1.55 & 1.04 & 0.51 \\
S61 & M0.5 II & 10.47 & 0.68 & -0.15 & 0.83 \\
S7 & B3-4 III (b4) & 11.52 & 0.73 & -0.08 & 0.81 \\
S8 & B6-8 III (b5) & 10.63 & 0.73 & $\ldots$ & $\ldots$ \\
S9 & B3-4 IIIshell & 11.20 & 0.78 & -0.10 & 0.88 \\
S10 & B5-7 III-IV (b5) & 11.50 & 0.74 & -0.13 & 0.87 \\
S12 & B3-5 IV-V (b4) & 11.62 & 0.74 & -0.13 & 0.87 \\
S13 & B3-5 IV-V (b5) & 11.62 & -0.12 & 0.87 \\
S15 & B4-5 IV-V (b5) & 11.95 & 0.75 & -0.12 & 0.79 \\
S16 & B5-7 III-IV (b5) & 11.56 & 0.67 & & \\
\hline
\end{tabular}

Notes.

a The typical uncertainty of the photometry is 0.02 mag in $K_{\mathrm{S}}$ and $0.03 \mathrm{mag}$ in $\left(J-K_{\mathrm{S}}\right)$.

$\mathrm{b}$ The whole range of spectral types and luminosity classes compatible with the spectral information available is shown.

${ }^{c}$ Photometric spectral types are given in parentheses.

with the expectations for a such a supergiant, especially if it is moving redward for the first time. In view of this, we accept the morphological classification F8 Ib. This object must thus lie on the instability strip.

\subsubsection{Blue Stars}

Figure 7 shows spectra of stars with intrinsically blue spectra, while Figure 8 shows the spectra of two stars with emission lines. The brightest early-type objects are S7 and S9. The $z$ band is not rich in classification features for B-type stars (Andrillat et al. 1995; Munari \& Tomasella 1999). Moderately accurate spectral types can be achieved for supergiants, but stars close to the main sequence are poorer in spectral features and can only receive approximate classifications (Negueruela et al. 2010a). Apart from the strength and width of Paschen lines, the main criterion is the presence of He I lines, which are only moderately strong in supergiants and are hardly seen in mid- and late-main-sequence B-type stars. All the spectra displayed are clearly dominated by broad Paschen lines and are therefore B-type stars of low or moderate luminosity. The only exception is S9, which looks like an A-type supergiant. However, the presence of O I $8448 \AA$ in emission betrays this as a Be star. Some Be shell stars have absorption spectra (due to the circumstellar disk seen close to edge-on) that strongly resemble A-type supergiants (Andrillat et al. 1988).

Star S7 has very well-defined Paschen lines and resembles rather closely the B5 II star in Figure 7, though it has lower luminosity. The narrow O I $8448 \AA$ indicates a slow rotator and most likely a giant. This is confirmed by the sharpness of the Paschen lines (compare with the B3 V star). We estimate a spectral type B3-4 III. S16 is very similar, though it rotates faster or has a lower luminosity. S10 is a fast rotator, but is likely a giant as well, as a weak He I $8779 \AA$ A seems to be present. S12, $\mathrm{S} 13$, and $\mathrm{S} 15$ are closer to the main sequence. The fact that $\mathrm{O}$ I $8448 \AA$ is weak compared with Paschen 18 places them close to B3. The spectrum of S19 (Figure 8) is very noisy, but OI $8448 \AA$ is clearly in emission. Therefore, it is a Be star. According to 


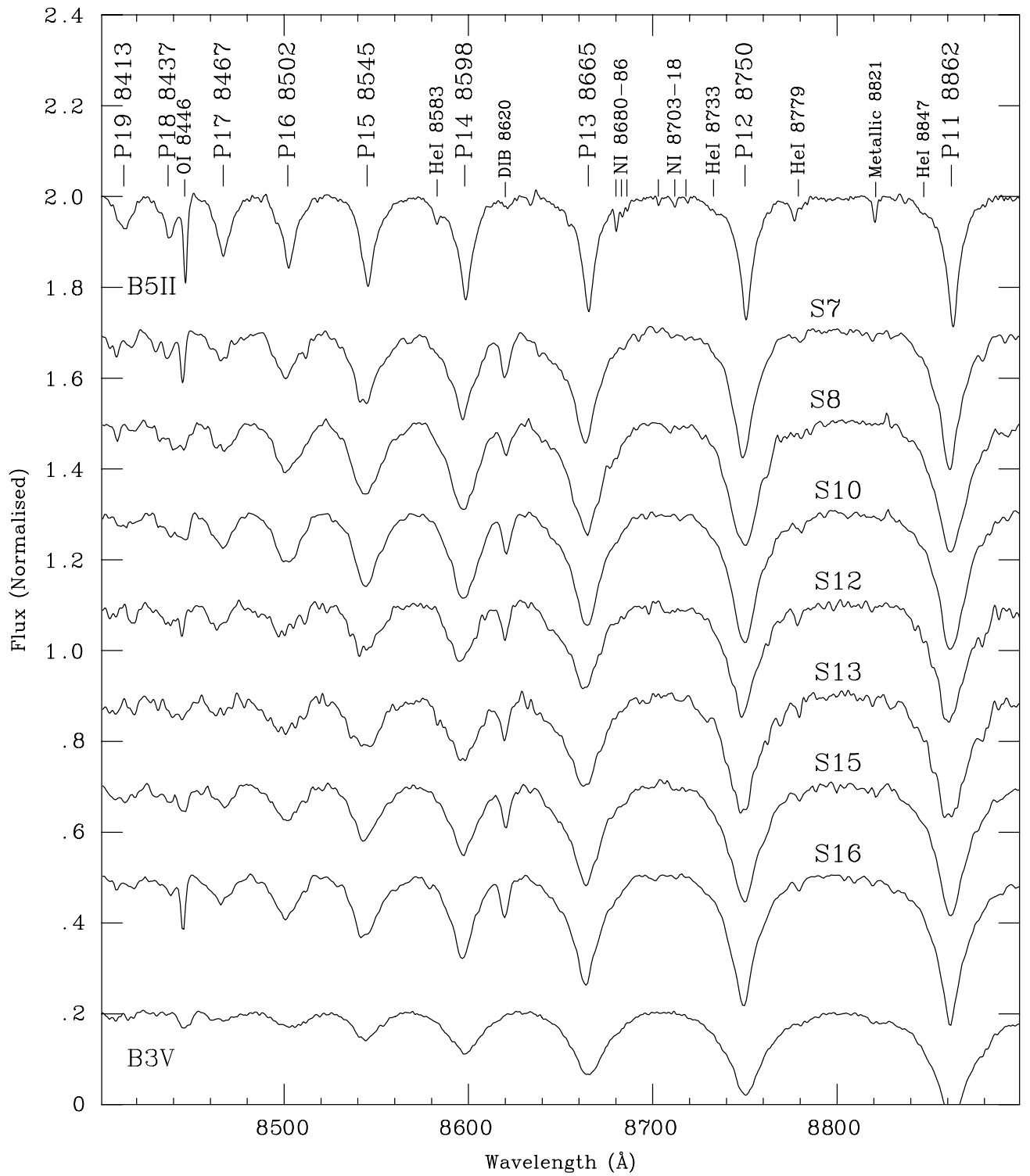

Figure 7. $z$-band spectra of a sample of intrinsically blue stars in Be 55, together with two reference stars from the Indo-US library (Valdes et al. 2004).

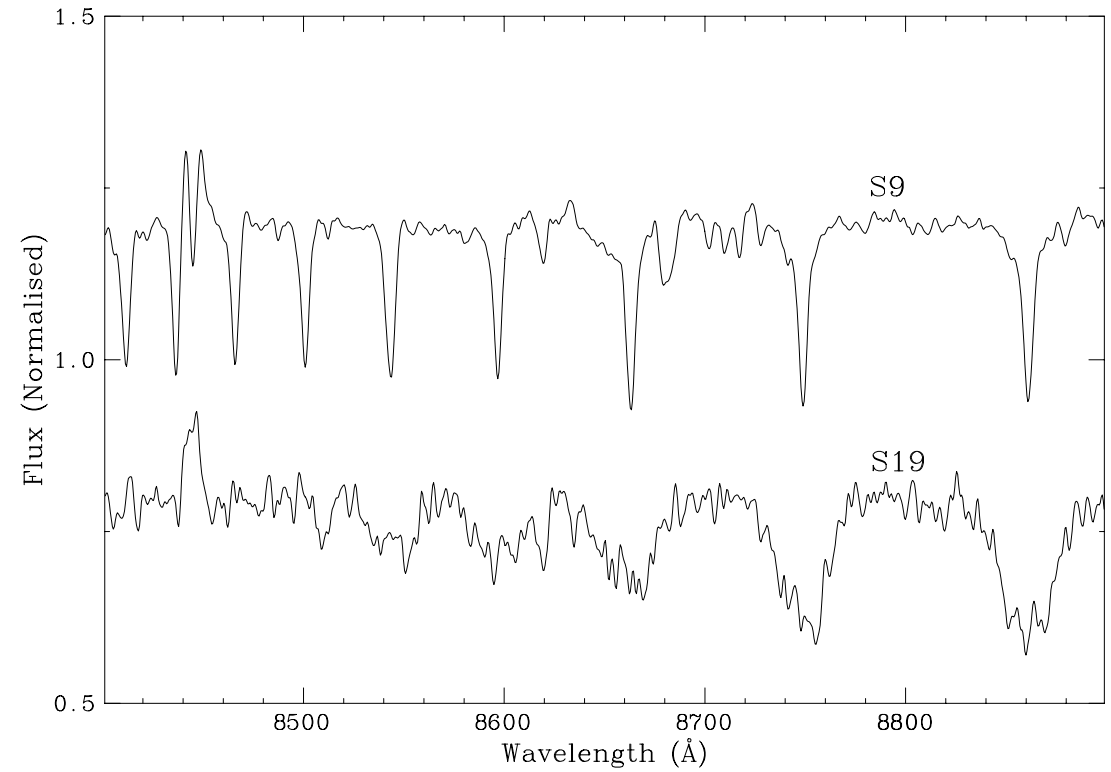

Figure 8. $z$-band spectra of two Be stars in Be 55. S9 is a shell star and the second brightest blue star in the cluster. 
Andrillat et al. (1988), late Be stars do not show this line in emission, but only filled-in. Therefore, this star is B5 Ve or earlier. Based on the same criterion and its brightness, S9 is likely to be a shell star of spectral type similar to S7. Finally, S8 looks later than all the others, though its reddening is similar to those of the other B stars. A summary of estimated spectral types is given in Table 7 .

Caron et al. (2003) developed a method to classify B-type stars by comparing the EWs of the Paschen lines. Application of those criteria suggests slightly later spectral types for our stars, though not all criteria suggest the same result (something expected, as they are only approximate). Following them, S7 would be $\sim$ B5 III, S8 and S10 would be $\sim$ B8 III, and the rest of the stars would be $\sim \mathrm{B} 5 \mathrm{~V}$. These small differences are in all likelihood due to the continuum determination, which is somewhat subjective, and in the case of the Paschen lines (with their very broad wings) may depend to some degree on signalto-noise ratio $(\mathrm{S} / \mathrm{N})$ and resolution.

\subsection{Infrared Extinction}

From the spectral types derived, we can calculate individual infrared reddenings. For the red (super)giants, we use the calibration of Straižys \& Lazauskaite (2009), which uses 2MASS colors. For the blue stars, we use the same calibration, assuming that giants have the same colors as main-sequence stars. For the F supergiant, we use the value from Koornneef (1983). The values derived are shown in Table 7 . The reddening seems to be moderately variable across the face of the cluster. The values for the supergiants show a higher dispersion than those of the blue stars, but the average reddening for the six red (super)giants (excluding the outlier S61) is $E\left(J-K_{S}\right)=0.89 \pm 0.07$ (the error is the standard deviation), while the average for seven B-type stars without emission lines is $E\left(J-K_{S}\right)=0.85 \pm 0.04$, fully compatible within their errors. Using the calibrations of Winkler (1997) or Wegner (1994) for the blue stars produces slightly different individual values, but compatible averages.

Comparison of the $E(B-V)$ (Table 5) and $E\left(J-K_{\mathrm{S}}\right)$ excesses for these objects (Table 7) confirms that the extinction law in this direction is close to the $R=3.1$ galactic average, for which $E(B-V) \approx 2 \times E(J-K)$. It is also interesting to note that the B-type stars spectroscopically observed consistently have $E(B-V)$ below the cluster average. This suggests that some of the faint late-B stars taken as photometric members could be background objects.

\subsection{Cluster Age}

The dereddened CMD for Be 55 (Figure 5) shows that stars brighter than $M_{V} \approx-1$ start deviating from the ZAMS. This absolute magnitude corresponds to spectral-type B4 V (e.g., Turner 1980), in good agreement with the determination of spectral types, which indicate that the brightest B-type stars are B3-6 III-IV objects. With such a turnoff, the cluster is expected to have an age $\gtrsim 40$ Myr. We can improve on this value by fitting isochrones in the different observational CMDs to the position of the red stars and the turnoff.

A fundamental issue is the correct determination of color excesses at such high reddenings. Figure 9 shows the observational $V /(U-B)$ diagram for the cluster together with isochrones for $\log t=7.6$ (40 Myr) and $\log t=7.7$ (50 Myr) from Marigo et al. (2008). Both isochrones fit well the position of the main sequence and the turnoff. S7 appears as a mild blue straggler, which is consistent with its spectral type (a B3-4 III giant when

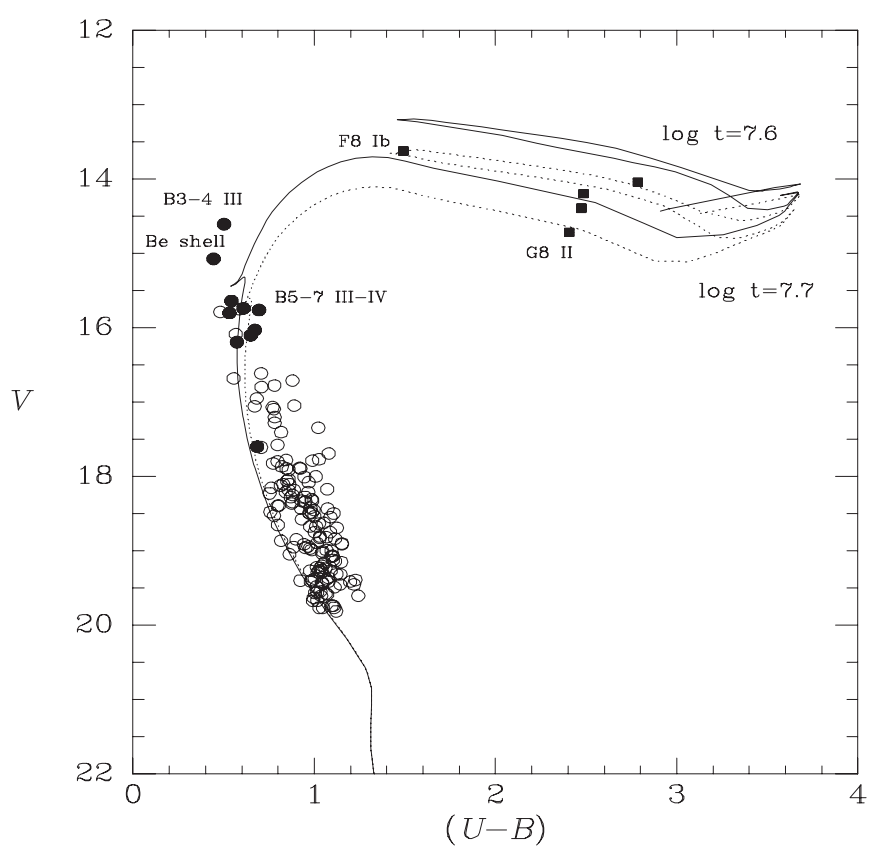

Figure 9. $V /(U-B)$ diagram for likely members of Berkeley 55. Filled circles are confirmed spectroscopic members, while open circles are likely (photometric) members. Two isochrones of Marigo et al. (2008) are shown, both reddened by $E(B-V)=1.85$ and displaced to $\mathrm{DM}=13.0$. The isochrones have been reddened taking into account color effects, following the procedure described in Girardi et al. (2008).

the turnoff is at B4 V). S9 may also be a blue straggler, but shell stars are known to have anomalous $(U-B)$ colors. The isochrones have been reddened following the procedure described in Girardi et al. (2008), which takes into account color effects for late-type stars. In spite of this, the position of the $\mathrm{K}$ (super)giants does not fit the red clump very well for any of the two isochrones. This is likely due to the difficulty in reproducing the extinction law with the polynomial approximation of Cardelli et al. (1988).

Figure 10 shows the $K_{\mathrm{S}} /\left(J-K_{\mathrm{S}}\right)$ CMD for cluster members, with photometry from 2MASS. Isochrones from Marigo et al. (2008) for the same ages are shown, reddened by $E\left(J-K_{\mathrm{S}}\right)=$ 0.85 , the value found for the blue spectroscopic members. All members selected from their $U B V$ colors fall to the right of the isochrones, confirming that this excess corresponds to the less reddened members. Some likely members seem to have much higher color excesses, suggesting that they are either Be stars or background B-type stars (though this is not generally supported by their photometric spectral types). In this CMD, the $\mathrm{K}$ (super)giants occupy positions in very good agreement with the 50 Myr isochrone.

The bright star S61 situated $\sim 4$. 7 from the cluster core falls together with the red (super)giants in this CMD. However, its position does not fit well with its spectral type, later than all the other evolved stars. As seen in Table 7, its reddening is rather lower than that of confirmed members. Its $(B-V)$ magnitude, taken from Maciejewski \& Niedzielski (2007), is compatible with the low reddening. Therefore, this object could be a foreground star as well as a cluster member with much lower reddening. This should be checked via radial velocity measurements. ${ }^{3}$

\footnotetext{
3 Even though our spectra were not taken to measure radial velocities, direct cross-correlation between the spectra of S1 and S61 (the two objects observed with the same configuration) suggests that their radial velocities are very similar.
} 


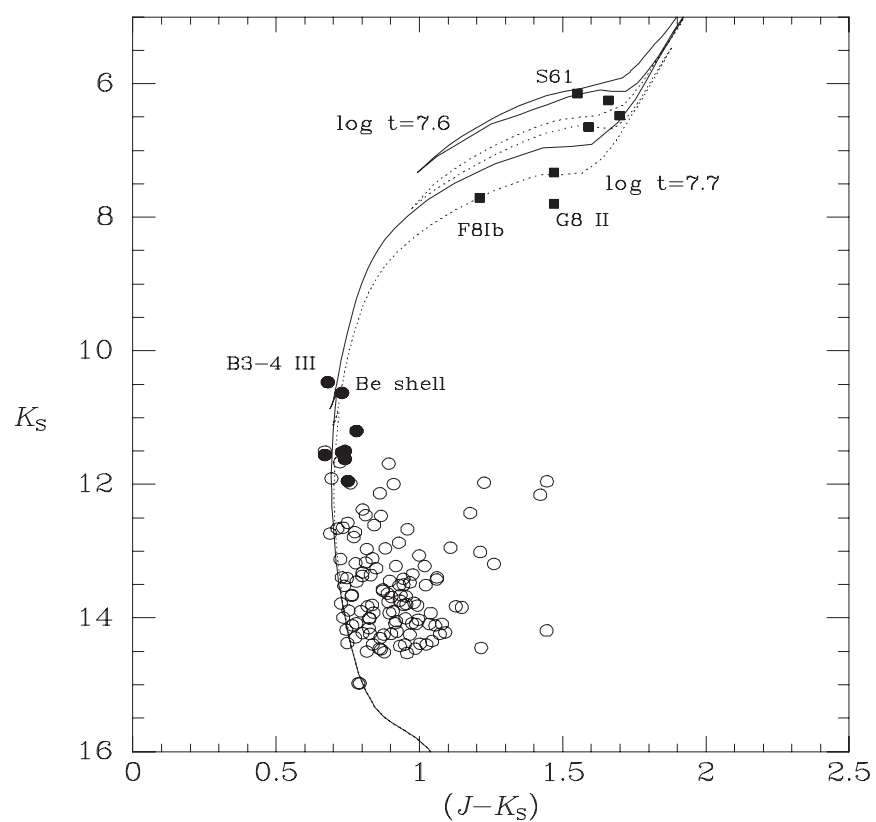

Figure 10. $K_{\mathrm{S}} /\left(J-K_{\mathrm{S}}\right)$ diagram for likely members of Berkeley 55. Filled circles are confirmed spectroscopic members, while open circles are likely (photometric) members. Two isochrones of Marigo et al. (2008) are shown, both reddened by $E\left(J-K_{\mathrm{S}}\right)=0.85$ and displaced to $\mathrm{DM}=13.0$.

\section{DISCUSSION}

We have presented spectroscopy of a sizable sample of stars in the open cluster Berkeley 55, revealing a population of seven low-luminosity supergiants or bright giants (one of them, not a certain member), four mid-B giants, and a main sequence starting around $\mathrm{B} 4 \mathrm{~V}$.

\subsection{Cluster Parameters}

Only two previous studies of the cluster have been published. Maciejewski \& Niedzielski (2007) derive an age of 315 Myr. Tadross (2008) used their result to calibrate his fitting procedure and therefore derives a similar age of $300 \mathrm{Myr}$, from a fit to the 2MASS data alone. This age is completely incompatible with the observed population, as stars earlier than B5 are only seen in clusters younger than 100 Myr. Clusters with $\sim 100$ Myr have stars of spectral type $\sim$ B6 around the main-sequence turnoff (e.g., NGC 7790, Matthews et al. 1995; Berkeley 58, Turner et al. 2008).

The difference between our age determination and that of Maciejewski \& Niedzielski (2007) is almost certainly due to the identification of the evolved population. The decontaminated $V /(B-V)$ in Maciejewski \& Niedzielski (2007) does not show a clump of red stars, as in our Figure 4 . Some of the (super)giants seem to be blended in their images and do not appear in their original photometry. The others are not conspicuous when a wide field is considered, because of contamination by field interlopers with similar $(B-V)$ color. The stars forming this clump, however, cannot be overlooked when the infrared data are considered. S1 is the brightest star in $K_{\mathrm{S}}$ within $10^{\prime}$ of the cluster center, and there are only five field stars with $K_{\mathrm{S}}$ magnitudes comparable to the seven red (super)giants. The seven candidate members form a clearly separated clump in the $\left(J-K_{\mathrm{S}}\right) / K_{\mathrm{S}}$, $H-K_{\mathrm{S}} / K_{\mathrm{S}}$, and $Q / K_{\mathrm{S}}$ diagrams and have similar (and unusual) spectral types. Though accurate radial velocities will be needed to confirm individual memberships, there can be no doubt that they represent the population of evolved stars in the cluster.
The small systematic offset in $(B-V)$ between our photometry and that of Maciejewski \& Niedzielski (2007) is unlikely to have biased their age determination. It is simply very difficult to obtain an accurate fit when photometry in only two bands is available.

At Galactic longitude $\ell=93.0$, a distance $\sim 4 \mathrm{kpc}$ (corresponding to our DM $=13.0$ ) seems compatible with tracers of the Perseus arm, though some Galactic models suggest a higher distance for this feature. Spiral tracers in this direction are scarce. NGC $7067(\ell=91.2)$ has distance estimates of $3.6 \mathrm{kpc}$ (Yadav \& Sagar 2004) and $4.4 \mathrm{kpc}$ (Hassan 1973). NGC $7128(\ell=97.3)$, with DM $=13.0 \pm 0.2(4 \mathrm{kpc})$, is another likely tracer at some distance from Be 55 (Balog et al. 2001).

As the age of Berkeley 55 is $\sim 50 \mathrm{Myr}$, the red stars in the cluster should have initial masses $M_{*} \gtrsim 7.0 M_{\odot}$ (Marigo et al. 2008), or slightly higher if they started their lives rotating quickly (cf. Meynet \& Maeder 2000). Such stars define the boundary between intermediate-mass and massive stars, and therefore can provide important constraints for stellar evolution models. Milky Way clusters with similar ages and rich populations of RSGs (or bright giants) are relatively scarce. NGC 6649 ( 50 Myr; Turner 1981) with one Cepheid and four red (super)giants is one of the few examples. NGC 6664 (also 50 Myr; Schmidt 1982) has a similar population, but is less well studied. As a consequence, the sample of Galactic RSGs studied by Levesque et al. (2005) contains almost no stars in the $6 M_{\odot}<M_{*}<10 M_{\odot}$ range. The presence of seven candidate evolved members makes Berkeley 55 a very suitable target to study the physical effects operating during the He-core burning phase of intermediate-mass stars.

\subsection{The Lowest Mass for a Supergiant}

As mentioned in the introduction, the separation between red supergiants and red bright giants is morphological and does not coincide with the separation between high-mass and low-mass stars. Of course, making such a distinction necessarily implies dividing a continuum into artificial boxes. ${ }^{4}$ In spite of that, defining an age limit after which clusters do not contain RSGs seems interesting, as new clusters are being discovered where high extinction renders only the bright red stars accessible for spectroscopy (e.g., Negueruela et al. 2010b; Davies et al. 2011).

Looking at the lists of yellow evolved stars in clusters from Harris (1976) or Sowell (1984, 1987), it is difficult to set an age limit after which Ib supergiants are not seen. A few objects have been classified as $\mathrm{K} \mathrm{Ib}$ supergiants in clusters $\gtrsim 100$ Myr old, but in all cases there are alternative lower-luminosity classifications based on more recent data (for example, star 164 in NGC 129, classified K2.5 II-III by Keenan \& McNeil 19895; or star 110 in NGC 2516, classified M1.5 IIa by Keenan \& Pitts 1985). Most classical Cepheids appear as F/G Ib supergiants. Many clusters containing Cepheids are sparsely populated and have the Cepheid as only supergiant (e.g., Turner 1996). In clusters with larger populations, the ratio of $\mathrm{F} / \mathrm{G}$ supergiants to later spectral types is very variable. For example, NGC 7790 contains three F-type Cepheids, but no red (super)giants, while NGC 6067 (with two Cepheid members) contains a large number of red giants and supergiants (Mermilliod 1987).

\footnotetext{
4 It should be noted that many authors classically count luminosity class II objects as supergiants.

5 This star seems not to be a member, based on its radial velocity (Mermilliod et al. 2008), though it was considered a member for a long time (see references in Turner 1992).
} 
If we consider a number of clusters for which accurate ages in the range of interest have been derived through careful photometric analysis or equivalent methods, we find by decreasing age that NGC 2516 ( 140 Myr), which has no Cepheids, contains four bright red giants, with luminosity classes II or III (Keenan \& Pitts 1985; González \& Lapasset 2000). IC 4725, which is $\sim 90$ Myr old, contains one Cepheid supergiant and two class II red giants (Keenan \& Pitts 1985; Mermilliod 1987). Berkeley 82 with an age $\sim 70 \mathrm{Myr}$ contains two yellow bright stars with spectral types G2 II/Ib and K2 II (Forbes 1986), while Harvard 20 contains a G5 Ib supergiant at $\sim 60 \mathrm{Myr}$ (Turner 1980). On the other hand, NGC 6664, with an age $~ 50 \mathrm{Myr}$, contains several objects classified as bright giants by Harris (1976). Finally, NGC 6067 contains red stars with luminosity classes II and Ib, leading Mermilliod (1987) to doubt its estimated age $\gtrsim 100 \mathrm{Myr}$, which is also in contradiction with the observed period of its fundamental-mode Cepheid member V340 Nor.

In view of this, it seems that red stars close to the clump position are classified as $\mathrm{K}$ supergiants in clusters up to 50-60 Myr, while some stars are classified as such in somewhat older clusters. No cluster older than $\sim 100$ Myr seems to contain a bona fide K supergiant. Stars with F and G spectral types, such as Cepheids, are classified as supergiants at older ages, e.g., SZ Tau (F5-9 Ib) in NGC 1647 ( 150 Myr; Turner 1992).

The classifications derived for stars in Be 55 seem to confirm these trends, with stars on both sides of the divide at an age $\approx 50 \mathrm{Myr}$.

This research is partially supported by the Spanish Ministerio de Ciencia e Innovación (MICINN) under grants AYA200806166-C03-03, AYA2010-21697-C05-05, and CSD2006-70, and by the Generalitat Valenciana (ACOMP/2010/259).

The WHT is operated on the island of La Palma by the Isaac Newton Group in the Spanish Observatorio del Roque de Los Muchachos of the Instituto de Astrofísica de Canarias. The 2007 July observations were taken as part of the service program (program SW2007A63).

Based on observations made with the Nordic Optical Telescope, operated on the island of La Palma jointly by Denmark, Finland, Iceland, Norway, and Sweden, in the Spanish Observatorio del Roque de los Muchachos of the Instituto de Astrofísica de Canarias.

Some of the data presented here have been taken using ALFOSC, which is owned by the Instituto de Astrofisica de Andalucia (IAA) and operated at the Nordic Optical Telescope under agreement between IAA and the NBIfAFG of the Astronomical Observatory of Copenhagen

This research has made use of the Simbad database, operated at CDS, Strasbourg (France) and of the WEBDA database, operated at the Institute for Astronomy of the University of Vienna. This publication makes use of data products from the Two Micron All Sky Survey, which is a joint project of the University of Massachusetts and the Infrared Processing and Analysis Center/California Institute of Technology, funded by the National Aeronautics and Space Administration and the National Science Foundation.

\section{Facilities: ING:Herschel(ISIS), NOT (ALFOSC)}

\section{REFERENCES}

Andrillat, Y., Jaschek, C., \& Jaschek, M. 1995, A\&AS, 112, 475

Andrillat, Y., Jaschek, M., \& Jaschek, C. 1988, A\&AS, 72, 129

Arellano Ferro, A., Giridhar, S., \& Rojo Arellano, E. 2003, RevMexAA, 39, 3
Balog, Z., Delgado, A. J., Moitinho, A., et al. 2001, MNRAS, 323, 872

Cardelli, J. A., Clayton, G. C., \& Mathis, J. S. 1988, ApJ, 329, L33

Caron, G., Moffat, A. F. J., St-Louis, N., Wade, G. A., \& Lester, J. B. 2003, AJ, 126,1415

Carquillat, J. M., Jaschek, C., Jaschek, M., \& Ginestet, N. 1997, A\&AS, 123, 5

Cenarro, A. J., Cardiel, N., Gorgas, J., et al. 2001, MNRAS, 326, 959

Chiosi, C., Bertelli, G., \& Bressan, A. 1992, ARA\&A, 30, 235

Comerón, F., \& Pasquali, A. 2005, A\&A, 430, 541

Davies, B., Bastian, N., Gieles, M., et al. 2011, MNRAS, 411, 1386

Díaz, A. I., Terlevich, E., \& Terlevich, R. 1989, MNRAS, 239, 325

Draper, P. W., Taylor, M., \& Allan, A. 2000, Starlink User Note 139.12, R.A.L. Fitzgerald, M. P. 1970, A\&A, 4, 234

Forbes, D. 1986, PASP, 98, 218

Ginestet, N., Carquillat, J. M., Jaschek, M., \& Jaschek, C. 1994, A\&AS, 108, 359

Girardi, L., Dalcanton, J., Williams, B., et al. 2008, PASP, 120, 583

González, J. F., \& Lapasset, E. 2000, AJ, 119, 2296

Harris, G. L. H. 1976, ApJS, 30, 451

Hassan, S. M. 1973, A\&AS, 9, 261

Howarth, I., Murray, J., Mills, D., \& Berry, D. S. 1998, Starlink User Note 50.21, R.A.L.

Jeffries, R. D. 1997, MNRAS, 288, 585

Johnson, H. L., \& Morgan, W. W. 1952, ApJ, 117, 313

Keenan, P. C., \& McNeil, R. C. 1989, ApJS, 71, 245

Keenan, P. C., \& Pitts, R. E. 1985, PASP, 97, 297

Koornneef, J. 1983, A\&A, 128, 84

Landolt, A. U. 1992, AJ, 104, 340

Levesque, E. M., Massey, P., Olsen, K. A. G., et al. 2005, ApJ, 628, 973

Maciejewski, G., \& Niedzielski, A. 2007, A\&A, 467, 1065

Maíz-Apellániz, J. 2004, PASP, 116, 859

Mallik, S. V. 1997, A\&AS, 124, 359

Marigo, P., Girardi, L., Bressan, A., et al. 2008, A\&A, 482, 883

Matthews, J. M., Gieren, W. P., Mermilliod, J.-C., \& Welch, D. L. 1995, AJ, 110,2280

Mermilliod, J.-C. 1981, A\&A, 97, 235

Mermilliod, J.-C. 1987, A\&AS, 70, 389

Mermilliod, J.-C., Mayor, M., \& Udry, S. 2008, A\&A, 485, 303

Meynet, G., \& Maeder, A. 2000, A\&A, 361, 101

Mowlavi, N., \& Forestini, M. 1994, A\&A, 282, 843

Munari, U., \& Tomasella, L. 1999, A\&AS, 137, 521

Negueruela, I., Clark, J. S., \& Ritchie, B. W. 2010a, A\&A, 516, A78

Negueruela, I., González-Fernández, C., Marco, A., \& Clark, J. S. 2011, A\&A, 528, A59

Negueruela, I., González-Fernández, C., Marco, A., Clark, J. S., \& Martínez-Núñez, S. 2010b, A\&A, 513, A74

Negueruela, I., Marco, A., Israel, G. L., \& Bernabeu, G. 2007, A\&A, 471, 485

Negueruela, I., \& Schurch, M. P. E. 2007, A\&A, 461, 431

Poelarends, A. J. T., Herwig, F., Langer, N., \& Heger, A. 2008, ApJ, 675, 614

Salasnich, B., Bressan, A., \& Chiosi, C. 1999, A\&A, 342, 131

Schmidt, E. G. 1982, AJ, 87, 1197

Schmidt-Kaler, T. 1982, in Landolt-Börnstein, New Series VI, Vol. 2b (Berlin: Springer-Verlag)

Shortridge, K., et al. 2004, Starlink User Note 86.21, R.A.L.

Skrutskie, M. F., Cutri, R. M., Stiening, R., et al. 2006, AJ, 131, 1163

Smartt, S. J. 2009, ARA\&A, 47, 63

Smartt, S. J., Eldridge, J. J., Crockett, R. M., \& Maund, J. R. 2009, MNRAS, 395, 1409

Smith, N., Li, W., Filippenko, A. V., \& Chornock, R. 2011, MNRAS, 412, 1522

Sowell, J. R. 1984, ApJS, 55, 455

Sowell, J. R. 1987, ApJS, 64, 241

Stetson, P. B. 1987, PASP, 99, 191

Straižys, V., \& Lazauskaitè, R. 2009, Balt. Astron., 18, 19

Tadross, A. L. 2008, MNRAS, 389, 285

Turner, D. G. 1980, PASP, 92, 840

Turner, D. G. 1981, AJ, 86, 222

Turner, D. G. 1992, AJ, 104, 1865

Turner, D. G. 1996, J. R. Astron. Soc. Can., 90, 82

Turner, D. G., Forbes, D., English, D., et al. 2008, MNRAS, 388, 444

Valdes, F., Gupta, R., Rose, J. A., Singh, H. P., \& Bell, D. J. 2004, ApJS, 152 , 251

Wegner, W. 1994, MNRAS, 270, 229

Weidemann, V. 2000, A\&A, 363, 647

Winkler, H. 1997, MNRAS, 287, 481

Yadav, R. K. S., \& Sagar, R. 2004, MNRAS, 349, 1481

Zhou, X. 1991, A\&A, 248, 367 